Disclosures D. Pujara: None. H. Kamal: None. O. Mir: None. S. Reddy: None. K. Parsha: None. S. Patil: None. B. Gogia: None. P. Rai: None. C. Sitton: None. M. Chen: None. M. Abraham: None. M. Hussain: None. A. Hassan: None. A. Sarraj: None.

\section{P-030 VOXEL-BASED CALCULATIONS OF INTRASACCULAR ANEURYSM AND DEVICE VOLUME FILL}

S Schwartz*, B Fennell, C Settanni, N Norris, T Becker. Bioengineering Program, Mechanical Engineering, Northern Arizona Univeristy, Flagstaff, $A Z$

10.1136/neurintsurg-2021-SNIS.66

Introduction Various embolization techniques are available for intracranial aneurysms. The precise volume measurement of an aneurysm sac can help improve embolization techniques, such as: coils, liquid embolics, flow disruptors, and flow diverters. Aneurysm sizing and volumetric information help interventionalists assess flow and stability pre-treatment and potential remnant or recanalization risks post-treatment.

Materials and Methods This research project applies voxelbased volume calculations, from CT or MRI medical imaging data, to determine accurate 3-D aneurysm volume calculations. Additionally, the application can display clinically relevant parameters, such as aneurysm neck diameter, dome height and midline-dome width (for dome:neck (D:N) ratio calculations). To develop the measurement techniques, wide-neck canine aneurysm models $(n=10)$ were scanned with a Siemens Inveon Micro-CT scanner (University of Arizona TBIR, Tucson, AZ) and analyzed with InVesalius 3.0 software (Ministry of Health, Brazil).

The software loads the imaging data, discretizes the intrasaccular aneurysm, separating the device volume from the aneurysm sac. This data was compared to physical measurements of excised aneurysms and calibrated 2-D angiographic images.

Results Invesalius image processing techniques helped determine the domain of aneurysms with less than $5 \%$ volumetric error, when compared to measurements recorded during the aneurysm creation surgery. Current measurement techniques in 2-D planes have errors as high as 30\%. This technique helps determine the macroscopic properties of aneurysms, as well as the volumes of abnormal aneurysm shapes. The software was also used to measure $\%$ fill of a liquid embolic (NeuroCURE) delivered to the 10 canine aneurysms. In all 10 cases the precise delivery of NeuroCURE resulted in 90$100 \%$ aneurysm sac filling, versus $<30 \%$ for traditional coiling techniques.

Conclusion As new aneurysm treatment devices are developed and greater\% fill of the aneurysm sac is attempted, the need for precise aneurysm volume calculations will be of high priority. This project brings together clinical and engineering expertise to translate medical imaging data directly into volumetric measurements with highly precise calculations that are currently not available from 2-D angiographic images. Measuring the size and dimensional properties of aneurysms with voxel-based volume calculations provides a fast, reliable and repeatable resource for aneurysm assessment. This technique will help interventionalists appropriately assess risk and treatment options for a broad range of aneurysm morphologies.
Disclosures S. Schwartz: 1; C; 5R42NS097069-03 (NIH Grant\#)). B. Fennell: 1; C; 5R42NS097069-03 (NIH Grant \#). C. Settanni: 1; C; 5R42NS097069-03 (NIH Grant \#). N. Norris: 1; C; 5R42NS097069-03 (NIH Grant \#). T. Becker: 1; C; 5R42NS097069-03 (NIH Grant \#).

\section{P-031 EFFECT OF COVID-19 ON ACUTE ISCHEMIC STROKE: POPULATION-LEVEL EXPERIENCE}

Y Kim*, S Khose, S Salazar-Marioni, R Abdelkhaleq, S Sheth. Department of Neurology, UTHealth MGovern Medical School, Houston, TX

10.1136/neurintsurg-2021-SNIS.67

Introduction Several studies report substantial decreases in the volume of acute ischemic stroke (AIS) during the early stage of coronavirus disease 2019 (COVID-19) pandemic. Differences in characteristics of strokes such as increasing rates of large vessel occlusion (LVO) stroke were also observed comparing between pre-COVID and COVID eras. However, these findings were often based on data from selected hospitals or networks and estimates may be biased by level of stroke care and baseline case mix. A population-based study including rural hospitals and non-stroke centers covering a longer observation period beyond the early-pandemic epoch may lead to more accurate insights.

Methods We conducted a retrospective population-based study using the Texas Inpatient Public Use Data File, capturing all discharges from hospitals in the State of Texas excluding federal hospitals. We assessed quarterly changes in volume, characteristics, care of AIS, and patient outcomes from April 1, 2019-June 30, 2020 and compared two time periods. 2020Q2 was defined as the pandemic period and the previous one year as the pre-pandemic period. Cases were defined as inpatient hospitalizations to acute care hospitals with a primary diagnosis of AIS. ICD-10 diagnosis and procedure codes were used to identify AIS, LVO, use of intravenous tissue plasminogen activator (IV-tPA) and endovascular treatment (EVT). Hospitals were determined as CSC (Comprehensive Stroke Center)

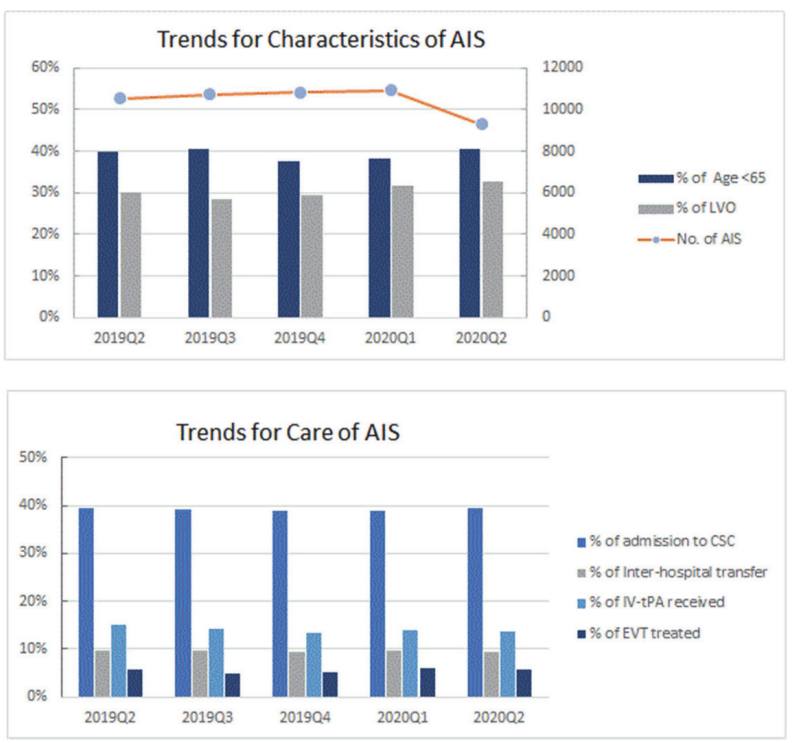

Abstact P-031 Figure 1 
and non-CSC based on designation by the Texas Department of State Health Services.

Results The number of AIS cases dropped substantially from 10,745 per quarter in the pre-pandemic period to 9,277 in the pandemic period $(-14 \%)$ in Texas. The percentage of patients aged younger than $65(39.0 \%$ vs $40.4 \%, \mathrm{p}=0.014)$ and LVO stroke $(30.0 \%$ vs $32.7 \%, \quad \mathrm{p}<0.001)$ slightly increased. Percentages of admission to CSC $(39.1 \%$ vs $39.5 \%, \mathrm{p}=0.57)$ and admission through inter-hospital transfer $(9.6 \%$ vs $9.3 \%, p=0.35)$ remained similar between pre-pandemic and pandemic periods. There was also no significant change in the use of IV-tPA $(14.1 \%$ vs $13.6 \%, \mathrm{P}=0.20)$ or EVT (5.4\% VS 5.8\%, P=0.12). In-hospital mortality slightly increased from $2.9 \%$ to $3.2 \%$ but it was not statistically significant $(p=0.13)$. However, the percentage of death or hospice discharge increased from $7.6 \%$ to $8.4 \%(p=0.003)$ and age and LVO type adjusted odds ratio was 1.13 (95\% CI 1.04-1.23).

Conclusion In this population-level study, significant decreases in AIS volume were observed with slightly higher incidence in younger population and higher LVO strokes. Rates of thrombolysis and thrombectomy remained unchanged but patients appeared to have worse outcomes.

Disclosures Y. Kim: None. S. Khose: None. S. Salazar-Marioni: None. R. Abdelkhaleq: None. S. Sheth: None.

\section{P-032 DISPARITIES IN STROKE: INFLUENCE OF SOCIOECONOMIC STATUS AND RACE ON TIMELY ACCESS TO MECHANICAL THROMBECTOMY}

AVT Nguyen, GM Cortez, M Baretta, A Aghaebrahim, E Sauvageau, R Hanel* Neurosurgery, Baptist Health System- Jacksonville, Jacksonville, FL

\subsection{6/neurintsurg-2021-SNIS.68}

Objective The present study aims to explore the influence of socioeconomic status (SES) and driving distance on timely access to mechanical thrombectomy.

Methods Patient data was collected retrospectively between 2016 and 2020 from a prospectively maintained database. Patients transferred from other facilities with driving distances of over 100 miles and onset-to-arrival (OTA) time and onset-to-puncture (OTP) time over 24 hours were excluded. Patient-level data included age, race, gender, median income based on zip code, driving distance, baseline dependency (mRS >2), baseline NIHSS scores, and wake-up stroke $\mathrm{y} / \mathrm{n}$. The driving distance was calculated using the Google Maps API, mapping distance between the center point of a zip code and the hospital's address. SES was defined by the zip code median income data from the 2019 U.S. Census Bureau's American Community Survey (ACS) 5-year Projection and patients' health insurance coverage. Primary outcomes OTA and OTP times, which were calculated by subtracting last-known-well time from arrival time and puncture time. We used a combination of nonparametric and multiple regression analyses to determine the predictors of outcome.

Results Among 305 patients collected, OTA time ranged from 9 to 1349 minutes, while OTP time ranged from 57 to 1422 minutes. Results demonstrated that race $(p<0.001)$, baseline NIHSS scores $(p<0.001)$, wake-up stroke $(p<0.001)$, and baseline mRS scores $(p<0.001)$ were all significantly associated with OTA and OTP time. Driving distance was significantly associated with OTA time $(\mathrm{p}=0.039)$ and OTP time $(p=0.005)$ as well. Median income, age, and gender did not exhibit any significant correlations.

Conclusions Race demonstrated to be an independent predictor of timely access to a stroke care facility and mechanical thrombectomy, while SES was not. Additional independent predictors were driving distance, wake-up stroke, and baseline functionality.

Disclosures A. V. T. Nguyen: None. G. M Cortez: None. M. Baretta: None. A. Aghaebrahim: 6; C; Advisory board for Rapid Medical. E. Sauvageau: None. R. Hanel: 2; C; Medtronic, Stryker, Cerenovous, Microvention, Balt, Phenox, Rapid Medical, Q'Apel. 4; C; InNeuroCo, Cerebrotech, eLum, Endostream, Three Rivers Medical Inc, Scientia, RisT, BlinkTBI, Corindus. 6; C; advisory board for MiVI, advisory board for eLum, dvisory board for Three Rivers, advisory board for Shape Medical, advisory board Corindus.

\section{P-033 DISPARITIES IN STROKE: ASSOCIATING PATIENT-LEVEL DEMOGRAPHIC FACTORS WITH LOSS OF FOLLOW-UP}

A Nguyen*, GM Cortez, M Baretta, A Aghaebrahim, E Sauvageau, R Hanel. Neurosurgery, Baptist Health System- Jacksonville, Jacksonville, FL

\subsection{6/neurintsurg-2021-SNIS.69}

Objective Loss of follow-up is a common barrier to achieving meaningful results in studies evaluating outcomes. In addition, lack of follow-up undermines the continuity of care that is crucial for ischemic stroke patients. Therefore, this study aims to explore feasible predictors and determinants of loss of follow-up in the ischemic stroke population, including socioeconomic status (SES) and various patient-level demographic factors.

Methods A retrospective review of a prospectively maintained database was performed between 2016 and 2020. Patient-level data collected include gender, age, race, traditional risk factors of stroke, and modified Rankin Score (mRS) at discharge. We measured SES using a combination of the zip code median income data from the 2019 U.S. Census Bureau's American Community Survey (ACS) 5-year Projection and patient health insurance coverage. Correlation between the rates of follow-up and demographic factors was analyzed using multivariate analyses.

Results Among 783 patients collected, 507 (64.7\%) came back for a 90-day follow up and 276 (35.3\%) did not. Our analysis demonstrated that there is a significant correlation between rates of follow up and race $(\mathrm{p}<0.001)$, discharge mRS ( $\mathrm{p}=$ $0.001)$, median income ( $\mathrm{p}=0.0001)$, and health insurance status $(\mathrm{p}=0.02)$. On the other hand, gender $(\mathrm{p}=0.82)$ and age $(\mathrm{p}=0.81)$ showed no significant correlation to follow up compliance. Furthermore, traditional stroke risk factors also demonstrated no significant correlation.

Conclusions Race, discharge mRS, median income based on zip codes, and health insurance coverage are potential determinants of whether an ischemic stroke patient who underwent thrombectomy would comply with a 90-day follow-up.

Disclosures A. Nguyen: None. G. M. Cortez: None. M. Baretta: None. A. Aghaebrahim: None. E. Sauvageau: None. R. Hanel: None. 